\title{
Transmission final lenses in the HiPER laser fusion power plant: system design for temperature control
}

\author{
A.R. Paramo, F. Sordo, D. Garoz, B. Le Garrec, J.M. Perlado and A. Rivera
}

\begin{abstract}
The European laser fusion project HiPER is developing technologically feasible components for a laser fusion power plant with an evacuated dry wall chamber which is likely to operate with a shock ignition scheme and direct targets. One of the key components is the final optics. In this work, we consider silica transmission final lenses and address the major issues regarding the unavoidable neutron irradiation they must withstand. For pre-commercial power plants ( $150 \mathrm{MJ}$ target yield at $10 \mathrm{~Hz}) \mathrm{a}$ distance of $16 \mathrm{~m}$ between the final lenses and target leads to maximum lens temperatures within tolerable limits. However, a non-uniform steady-state temperature profile is a major concern because it is the origin of unacceptable aberrations that severely affect the target spots. We have devised an active intervention system based on a heat-transfer fluid to keep the temperature profile as smooth as possible. The main characteristics of the temperature control system are defined throughout this work and enable the operation of the plant, both for the start-up procedure and for normal operation.
\end{abstract}

\section{Introduction}

Currently, the National Ignition Facility (NIF) is devoting its efforts to the demonstration of fusion laser ignition [1]. Recent achievements are very promising for the development of laser fusion. However, technological developments are needed to reach the goal of building a power plant. Several projects are already working on this goal, notably, LIFE [2] in the US and HiPER [3] in Europe. In commercial fusion plants several new aspects will have to be considered, in particular those related to materials under irradiation. One of these problems is studied in this paper: the effect of neutron irradiation on the final lenses of HiPER.

The final optics assembly (FOA) must be protected from the $\alpha, \beta, \gamma$ particles, charged particles and neutrons stemming from the target explosions at the chamber centre. In fact, whatever the solution is, at least one optical component will be in the field of view of the radiation and the debris from the target. In order to avoid or minimize back reflected light or any kind of radiation from the target, it is necessary either to focus the beam with optical components far from the target (and so far a large focal length will be required), or to tighten the beam with a small pinhole (in the radiation shield made of concrete) somewhere close to the chamber wall.

The solution adopted in HiPER is the use of a cassette of lenses with or without debris shields. This design has been detailed in [3] and is shown in figure 1. With this design, a $4 \mathrm{~m}$ focal length gives a total distance between the target chamber centre and pinhole of $16 \mathrm{~m}$ because the lens is placed at a $2 f-2 f$ position (magnification $=-1$ ). The size of this lens is $0.75 \times 0.75 \mathrm{~m}^{2}$ and the clear aperture for the beam is $0.6 \times 0.6 \mathrm{~m}^{2}$.

An alternative to the cassette of lenses is the use of grazing incidence metallic mirrors (GIMM). GIMM have been designed and tested during the high average power laser program [4]. The real drawback of the metallic mirrors (apart of radiation-resistance considerations) is the distance from the target: $24 \mathrm{~m}$ to the focal point, which is three times longer than the NIF/LMJ focal length $(8 \mathrm{~m})$; so far it is almost impossible to reach the same beam pointing accuracy on the target [5].

The use of the cassette of lenses chosen for HiPER is analysed in this paper. Following previous studies $[6,7]$ we 


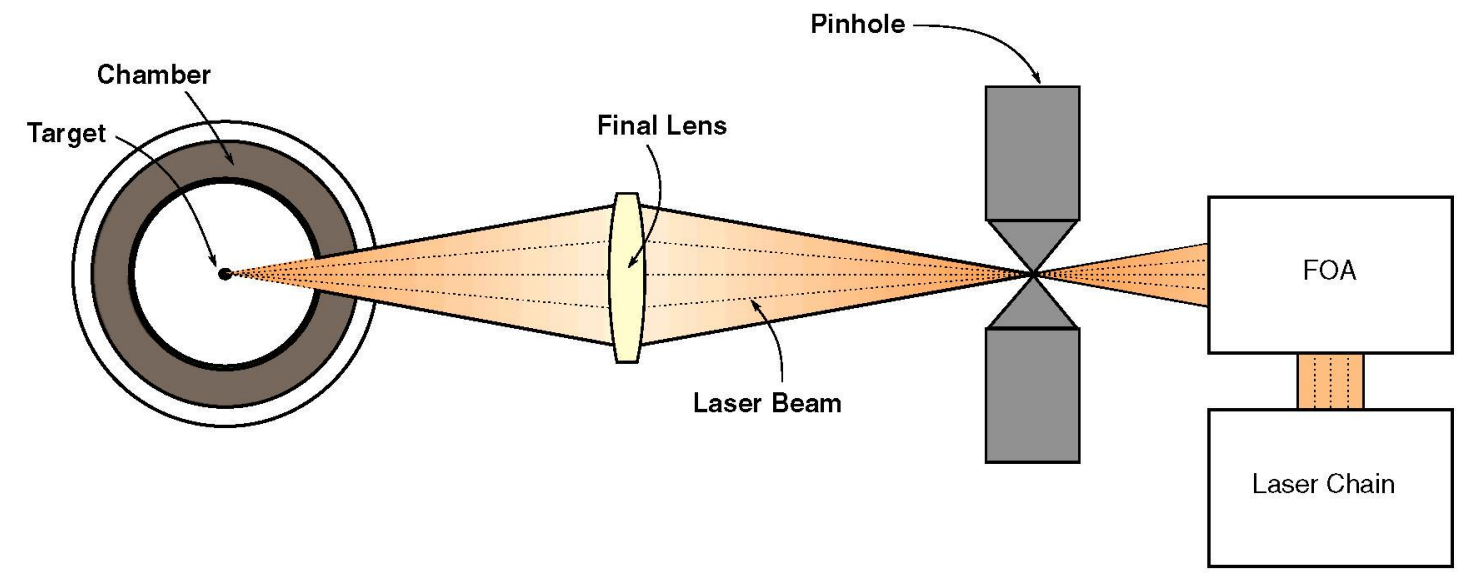

Figure 1. A schema showing the configuration of the final lens in the HiPER pre-commercial power plant. The laser is transported in the laser chain and passes through the FOA where it is smoothed and shaped. Then the laser passes through the pinhole that protects against irradiation and is finally focused onto the target by means of the final lens.

analyse problems related to neutron irradiation on the silica transmission final lenses, particularly the dependance of the lens focusing on temperature, both during the start-up and during normal operation. We show that a temperature control system is a must for proper lens performance and discuss several possibilities, concluding that the only realistic solution requires the use of a hot gas flow. Finally, we discuss the different design conditions and parameters used in this work. Although some parameters used in this study may differ from those finally used for the HiPER system, the purpose of this study is to provide a starting point for a realistic conceptual design that can be considered as a reference.

\section{Methodology}

\subsection{HiPER final lenses: design conditions}

In order to minimize risks, HiPER will be divided into different phases aimed to prove continuous mode operation in a laser fusion power plant. A pre-commercial power plant will produce an output power close to that of a commercial power plant, $\sim 1 \mathrm{GW}$. For this purpose high gain targets (yield around $150 \mathrm{MJ}$ ) should be designed, operating at high repetition rates $(\sim 10 \mathrm{~Hz})$. For the HiPER project, shock ignition, an advanced direct drive ignition scheme [8], is the preferred option. This scheme will use 48 laser beams to obtain a symmetric target illumination [9]. Each beam is also divided into a bundle of beamlets to produce a zooming effect [10]. The zooming effect is obtained with the arbitrary beam selection (ABS) system. It allows switching on and off the appropriate beamlets at each stage of the ignition scheme. Three types of beamlets are proposed in this work (see figure 2), for the different stages (foot, compression and shock). The beamlets are shaped and smoothed in the FOA, and then relayed through a pinhole of $\sim 4 \mathrm{~mm}$ diameter (see figure 1). Finally the beamlets are focused onto the deuterium-tritium (DT) target with the use of silica transmission lenses. We assume square biconvex lenses divided in a configuration of $4 \times 4$ quadrangles. Each lens quadrangle is used for a laser beamlet with the configuration shown in figure 2 . When each laser beamlet is focused onto the target they have a super-Gaussian profile, $I=I_{0} \cdot \exp \left(-(r / \Delta)^{m}\right)$, where $m=2, \Delta=0.62 \cdot R_{0}$ and
$R_{0}$ is $2,1.25$ or $0.5 \mathrm{~mm}$ for the foot, compression and shock beamlets, respectively. This choice for $R_{0}$ is based on an initial target radius of $2 \mathrm{~mm}$. Targets of around $2 \mathrm{~mm}$ are necessary for target gains of $150 \mathrm{MJ}$. The critical radius during the shock has been taken as $\sim 0.5 \mathrm{~mm}$ [9] and finally we have considered an intermediate size for the compression stage.

The dimensions of the final lenses are limited by laser damage. Damage in silica with nanosecond lasers appears for fluences higher than $10 \mathrm{~J} \mathrm{~cm}^{-2}$ [11]. For a target yield of $\sim 150 \mathrm{MJ}$, a laser shot of $\sim 1 \mathrm{MJ}$ (in 48 beams) is needed. This energy is deposited along the different stages (foot, compression and shock). The compression is the most demanding stage accounting for $75 \%$ [12] of the deposited laser energy. The remaining $25 \%$ will be mostly deposited during the shock stage. In order to keep the laser energy density below $10 \mathrm{~J} \mathrm{~cm}^{-2}$, the dimensions of the lens are $60 \times 60 \mathrm{~cm}^{2}$. With these dimensions, a laser fluence of $\sim 8.7 \mathrm{~J} \mathrm{~cm}^{-2}$ will be reached during the compression stage and $\sim 5.8 \mathrm{~J} \mathrm{~cm}^{-2}$ during the shock stage.

Once the lens dimensions are defined, it is important to address the lens distance to the target. The distance is first determined by the irradiation conditions and the maximum working temperature, assumed to be $1223 \mathrm{~K}$ [13]. We already pointed out in [6] that the intense ion pulses generated during the explosions of direct drive targets produce local melting with just one shot. Therefore, ion mitigation strategies must be adopted. Assuming full ion mitigation, the lenses are irradiated only by the fusion neutrons and $\mathrm{x}$-rays.

In [6] we studied the lens behaviour for a prototype plant with an output power of 50-500 MW (1-10 Hz, 50 MJ). Under these conditions it is possible to place the lenses at $8 \mathrm{mkeeping}$ their temperature below $1223 \mathrm{~K}$. However, in the case of precommercial conditions $(10 \mathrm{~Hz}, 150 \mathrm{MJ})$ the lens should be placed further than $8 \mathrm{~m}$ in order to keep the lens temperature below $1223 \mathrm{~K}$. Moreover, as discussed in this work, keeping the temperature below $1223 \mathrm{~K}$ is not the only condition necessary for the operation of the lens. In addition, it is necessary to keep a flat temperature profile along the lens and a constant temperature over time (section 3). Even making use of a sophisticated temperature control system based on a flow of gas (discussed in section 4), the most optimistic temperature 


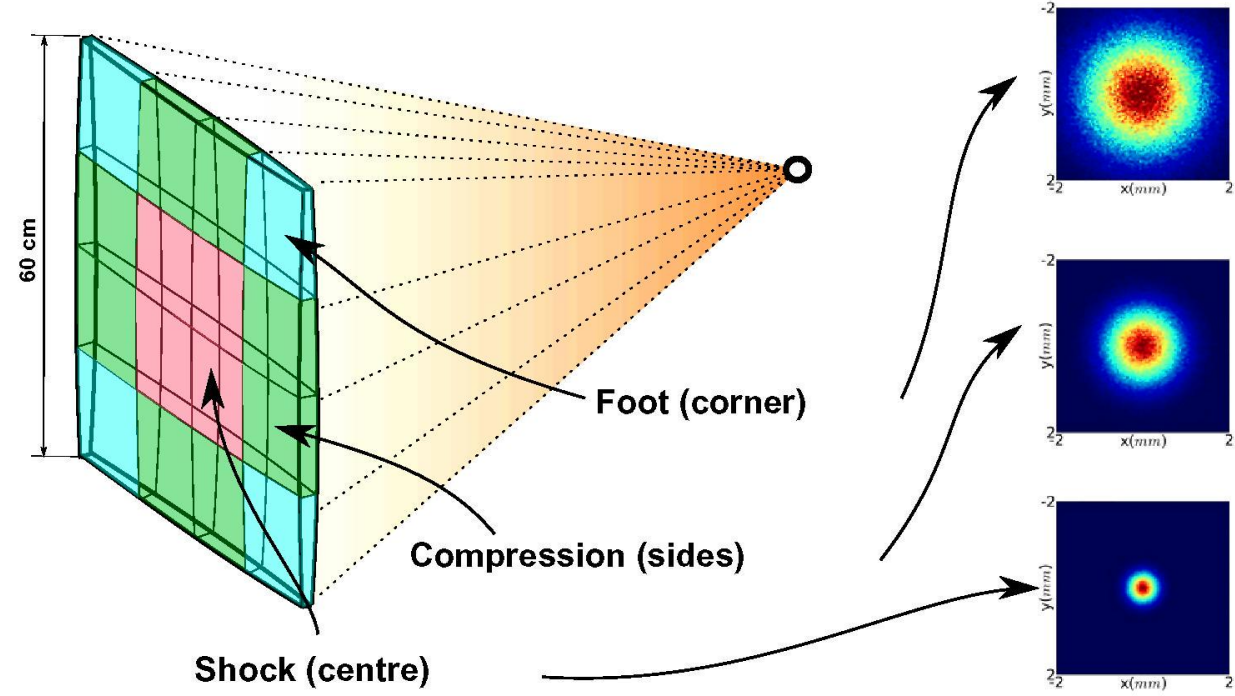

Figure 2. The final lens is divided in $4 \times 4$ quadrangles for the different laser beamlets. The foot beamlets pass through the four quadrangles of the corners (blue), the compression beamlets through the eight quadrangles of the sides (green) and the final shock beamlets through the four central quadrangles (red). The different laser beamlets are focused onto the DT target forming super-Gaussian profiles of different sizes (right).

profiles at $8 \mathrm{~m}$ turn out to be inappropriate (details are given in the appendix).

Assuming a realistic situation, i.e. biconvex transmission silica lenses, pre-commercial power plant conditions and direct drive targets, we must accept that the final lenses must be located far away from the targets. Therefore, in this work, we have set the lens position at $16 \mathrm{~m}$ from the targets. It will be shown that at this distance, it is possible to design a temperature control system that assures correct performance during all possible power plant operation regimes (startup, normal operation and special operation modes). Also, although outside the scope of this paper, it is interesting to note that the larger the distance between the final lens and target, the easier it turns out to be to implement an ion mitigation strategy, which will have to be mandatorily implemented for power plant operation.

Once the lens position is set, a number of important parameters can be calculated, such as the focal length, the lens thickness, the neutron flux and the neutron-generated light species in the lenses. We discussed in [14] the methodology to obtain these parameters and in table 1 there is a list of the design parameters employed in this work.

\subsection{Figures of merit}

An evaluation of the final lens optical performance is necessary to assess the validity of any possible final lens system. In particular, the effect of aberrations on the illumination uniformity must be addressed. A poor uniformity level will lead to instabilities during the target compression process.

The illumination uniformity level is quantified through its root mean deviation $(\sigma)$ :

$$
\sigma=\frac{\left[\int(I(\Omega)-\bar{I})^{2} \mathrm{~d} \Omega\right]^{1 / 2}}{\sqrt{4 \pi} \cdot \bar{I}}
$$

where $\bar{I}$ is the average intensity, $I$ the intensity at the target surface and $\Omega$ the integration variable.
Table 1. Design conditions for the HiPER final lenses. Irradiation conditions from [14].

\begin{tabular}{|c|c|}
\hline Parameter & Value \\
\hline Number of lasers & 48 \\
\hline Ignition scheme & Direct drive-shock ignition \\
\hline Target gain & $150 \mathrm{MJ}$ \\
\hline Repetition rate & $10 \mathrm{~Hz}$ \\
\hline $\begin{array}{l}\text { Beam profile } \\
\qquad I=I_{0} \cdot \exp \left(-(r / \Delta)^{m}\right)\end{array}$ & $m=2, \Delta=0.62 \times R_{0}$ \\
\hline $\begin{array}{l}\text { Target radius } R_{0} \\
\text { (foot, compression, shock) }\end{array}$ & $\begin{array}{l}2 \mathrm{~mm}, 1.25 \mathrm{~mm}, 0.5 \mathrm{~mm} \text {, } \\
\text { respectively }\end{array}$ \\
\hline Lens distance to chamber centre & $16 \mathrm{~m}$ \\
\hline Lens focal length & $8 \mathrm{~m}$ \\
\hline Lens size & $\begin{array}{l}60 \times 60 \mathrm{~cm}^{2}(4 \times 4 \text { quadrangles } \\
\left.\text { of } 15 \times 15 \mathrm{~cm}^{2}\right)\end{array}$ \\
\hline Maximum lens thickness & $28 \mathrm{~mm}$ \\
\hline Neutron flux in the lens & $2 \times 10^{17} \mathrm{~m}^{-2} \mathrm{~s}^{-1}$ \\
\hline $\begin{array}{l}\text { Averaged power density } \\
\text { due to neutrons }\end{array}$ & $510 \mathrm{~kW} \mathrm{~m}^{-3}$ \\
\hline Hydrogen production in the lens & 49 appm/fpy \\
\hline Helium production in the lens & 83 appm/fpy \\
\hline
\end{tabular}

To guarantee that the illumination uniformity is suitable for ignition, $\sigma$ should be kept below 1\% [15]. This requirement applies especially during the foot and compression stages because instabilities are formed in these stages. The illumination uniformity depends on several factors. The most important are geometrical restrictions (beam line configuration), system errors (pointing errors, target positioning) and the beam super-Gaussian profile. The illumination uniformity dependency on these factors was studied for HiPER in $[15,16]$. In this paper a new factor is introduced: the dependence of the illumination uniformity on the temperature of the final lens. Although the illumination uniformity $(\sigma)$ is the most important parameter, we have also studied the efficiency of laser deposition $(\eta)$. The efficiency is calculated as the ratio of power deposited in the target to the 
laser power

$$
\eta=\frac{\int I(\Omega) \mathrm{d} \Omega}{P_{0}}
$$

where $I$ is the laser intensity, $\Omega$ the integration variable in the target surface and $P_{0}$ is the laser power.

\subsection{Methodology}

Once the design conditions of HiPER are known (table 1) and the figures of merit defined, it is necessary to address the final lens performance. A multicode approach has been used for this purpose involving different fields such as neutronics, optics and fluid mechanics.

The neutron irradiation of the final optics has been calculated with MCNPX [17] using nuclear libraries ENDFVII [18]. The neutron source is obtained from the Aries project [19]. With the neutron dose, the temperature profile in the final lens has been calculated using Fluent [20] making use of the fluid properties obtained from the CoolProp database [21]. To retain the detail in the boundary layer, $2 \mathrm{D}$ simulations have been performed in different slices of the fluid channel using the $k \epsilon$ turbulence model. For the radiative model, the surface to surface (S2S) model is used.

For the evaluation of the optical performance, a ray-tracing code has been developed with a Monte Carlo approach. The ray-tracing code calculates the focal spot for one laser at each stage of the ignition scheme (foot, compression, shock) for a given lens temperature profile. Finally, the focal spot is projected into the spherical target with the 48 direct drive laser beams of the HiPER project.

\section{Temperature requirements}

\subsection{Temperature limits}

Due to neutron irradiation, colour centres are created in the final lenses $[14,22,23]$. As a result, optical absorption at $\sim 350 \mathrm{~nm}$ becomes appreciable. This is the typical laser wavelength for laser fusion facilities (the third harmonic of solid state lasers that use $\mathrm{Nd}$ as a dopant). Therefore, a loss of transparency in this band is undesired. Temperature plays a beneficial role, because colour centres efficiently anneal out at temperatures above $800 \mathrm{~K}$. Continuous operation for long periods of time relies on an efficient mechanism for colour centre annealing, thus the final lens systems must be designed to operate at a temperature higher than $800 \mathrm{~K}$. As mentioned above, the maximum working temperature is $1223 \mathrm{~K}$ [13]. Therefore, the final lens operation temperature must be within the range from 800 to $1223 \mathrm{~K}$.

\subsection{Temperature variation}

In addition, the final lens temperature must be kept constant during all the plant operation scenarios. This is due to the fact that the focal length varies with temperature. For a thin lens the focal length variation with temperature is shown in equation (3)

$$
\begin{aligned}
\frac{\partial f}{\partial T} & =\frac{\partial f}{\partial n} \cdot \frac{\partial n}{\partial T}+\frac{\partial f}{\partial R} \cdot \frac{\partial R}{\partial T}=-f\left(\frac{\partial n / \partial T}{n-1}+\alpha\right) \\
& \approx-f \cdot \frac{\partial n / \partial T}{n-1}
\end{aligned}
$$

where $f$ is the focal length, $T$ is the temperature, $n$ is the refractive index and $R$ is the lens curvature radius. The term $\frac{\partial n / \partial T}{n-1}$ is the variation of the focal length related to the variation of the refractive index and $\alpha$ is the material thermal expansion, which gives the variation of the focal length due to geometry variation. For the case of silica $\frac{\partial n / \partial T}{n-1} \sim 3 \times 10^{-5} \mathrm{~K}^{-1}$ [24], and $\alpha \sim 5 \times 10^{-7} \mathrm{~K}^{-1}$ [13], i.e. two orders of magnitude lower ${ }^{4}$.

For lenses located $16 \mathrm{~m}$ away from the target, deviations in temperature of $\sim 20 \mathrm{~K}$ induce variations of $\sim 5 \mathrm{~mm}$ in the lens focal length. The variation in the lens focal length will divert the laser focal spot. This deviation will be $\sim 0.4 \mathrm{~mm}$ for the foot beamlets, $\sim 0.3 \mathrm{~mm}$ for the compression beamlets and $\sim 0.1 \mathrm{~mm}$ for the shock beamlets. When we compare these deviations with the focal spot sizes, $2 \mathrm{~mm}, 1.25 \mathrm{~mm}$ and $0.5 \mathrm{~mm}$ for the foot, compression and shock stages, respectively, we observe that the deviation is significant and affects the energy deposition on the target.

Therefore, the lenses must be designed to operate at their design temperature $\left(T_{0}\right)$. This temperature must be maintained during the different operational regimes (start-up, partial regime or normal operation) and only small deviations are allowed.

\subsection{Temperature profile}

Biconvex lenses are thicker at their centre $(\sim 28 \mathrm{~mm}$ at $16 \mathrm{~m}$ from target) than at the sides $(\sim 5 \mathrm{~mm})$. This causes a higher energy deposition (from neutrons) at the lens centre than at the sides. Since the thermal conductivity of silica is very low, a steady-state non-uniform temperature profile is established along the radial dimensions of the lens. The result is a significantly higher temperature at the centre than at the sides. Different temperatures in the different parts of the lens (centre, sides and corners) cause optical aberrations.

An example is shown in figure 3 for the compression stage. Beamlets pass through the eight quadrangles of the sides. Figure 3(a) shows an ideal case with all the quadrangles at the same temperature. Each beamlet is focused on a super-Gaussian spot and the superposition of all the laser beamlets illuminates the target with a high uniformity level $(\sigma=0.16 \%)$. In figure $3(b)$ the beamlets pass through the quadrangles at a temperature $20 \mathrm{~K}$ higher than the design one $\left(T_{0}=950 \mathrm{~K}\right)$. The superposition of the 48 laser beams results in a larger spot and thus, the efficiency $(\eta)$ is slightly reduced, but the illumination uniformity level is still good $(\sigma=0.27 \%$ ). Finally, in figure 3(c) the beamlets pass through the quadrangles at different temperatures, half of them $20 \mathrm{~K}$ above the design temperature $\left(T_{0}=950 \mathrm{~K}\right)$ and the other half $20 \mathrm{~K}$ below $T_{0}$. The resulting spot is strongly deformed and not centred. Some regions of the target are overfocused and other underfocused. Due to the lack of symmetry in the temperature profile the uniformity level in the target is not suitable for ignition $(\sigma \gg 1 \%)$.

Summarizing section 3 , the temperature profile along silica transmission final lenses must be as flat as possible, constant during all the operation procedures and within certain limits, $800-1223 \mathrm{~K}$.

4 The focal distance is also affected by the lens weight. The deformation due to lens weight is $\epsilon \sim 10^{-7}$. This effect is similar to the thermal deformation $(\alpha \cdot \Delta T)$ and its influence on the focal distance is much lower that the variation of the refractive index due to temperature. 


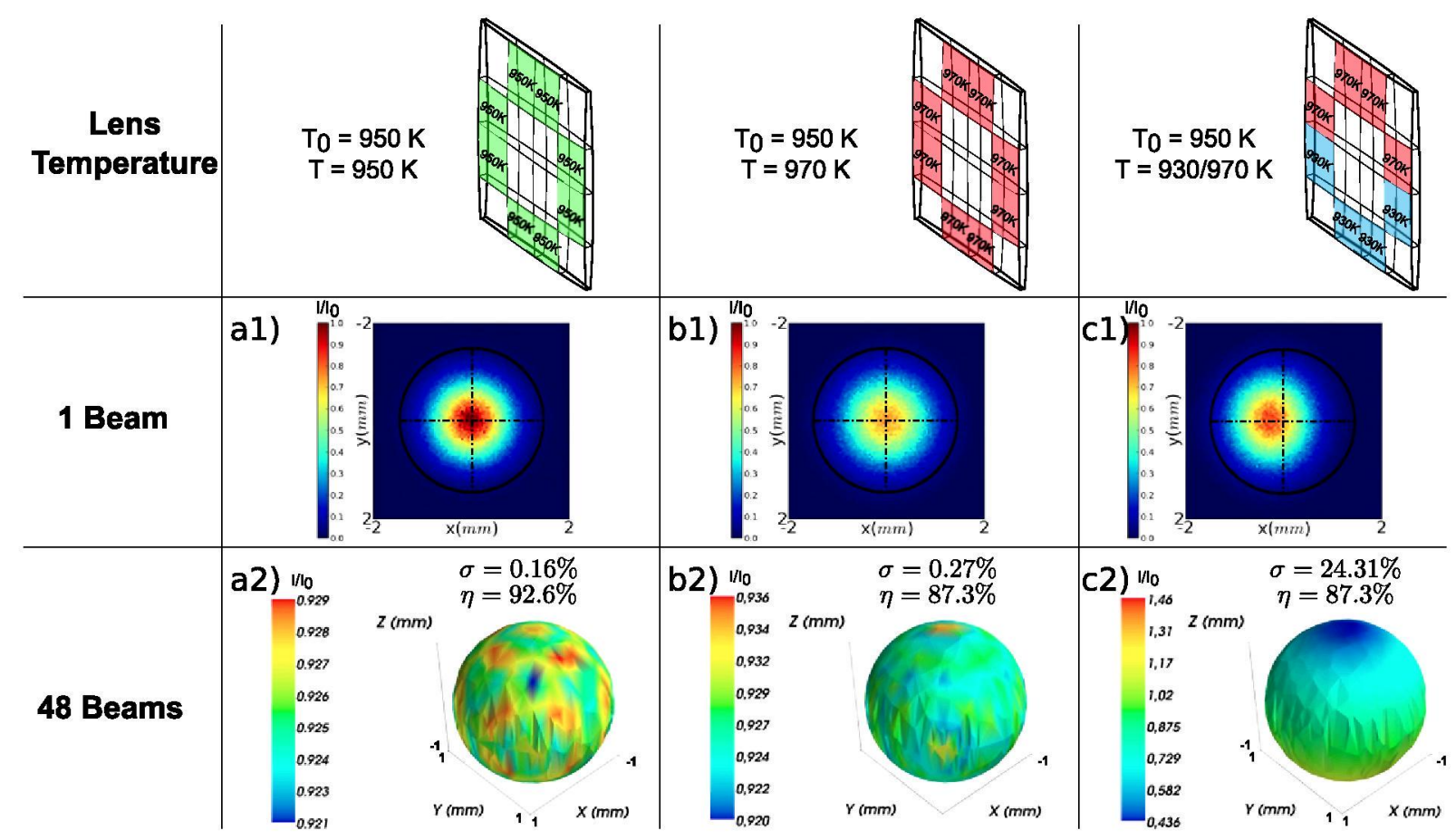

Figure 3. An example of the focal spot behaviour under different temperature variations. (Top) a schema of temperatures is shown (green for normal, red for hot and blue for cold). (Middle) the focal spot for one beamlet is shown. (Bottom) target illumination resulting from the superposition of 48 laser beams is shown. Several scenarios are shown. Note that for the laser deposition on the target (bottom) the scales are different.

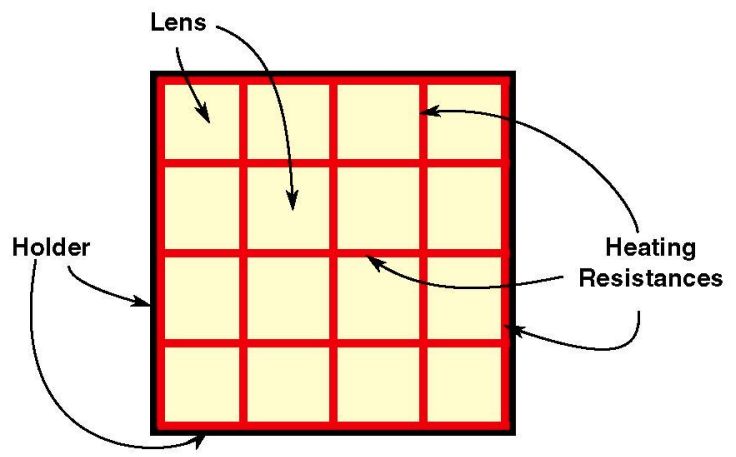

Figure 4. A temperature control system based on a heating holder and line resistances (red) along the surface of the lens.

\section{Temperature control system}

In order to fulfil the temperature requirements for the HiPER silica transmission final lenses, a temperature control system must be designed. We have studied the following systems: (a) no control system (i.e. lenses exposed to irradiation) just for comparison, (b) a control system based on heating resistances and (c) a control system based on a heat-transfer fluid.

A description of a system (b) based on heating resistances is given in figure 4 . The heating resistances are placed between the lens quadrangles and control the temperature at the quadrangle borders. The centre of the lens quadrangles should remain at an acceptable temperature thanks to heat conduction from the quadrangles borders. However, due to the low conductivity of silica the temperature gradient is high and temperature in the quadrangle centres is not effectively controlled. With a finer grid for the heating resistances the temperature could be controlled. But a finer grid is not a feasible solution as the laser would impact on the resistances with negative effects, and the complexity of the system would be greatly increased. Therefore, such a solution has been discarded.

In order to have better control of the temperature, a system (c) using a heat-transfer fluid is proposed with much better results. This system is shown in figure $5(c)$. The fluid will heat the whole lens surface resulting in a flat temperature profile. In the following paragraphs, the conditions to obtain a flat temperature profile as well as other design parameters are discussed.

In the system (c), the fluid is confined in two channels between the lens and silica-made windows. Due to the lens curvature, the channel width changes throughout the channel. Therefore in the flow pattern, the fluid velocity is higher in the middle of the channel than at the inlet/outlet. It is also important to point out that the fluid temperature changes over the channel. The fluid outlet temperature is lower than the inlet temperature. If a parallel flow pattern along both channels is chosen, the temperature profile would not be symmetric, decreasing the uniformity level in the focal spot, similar to the situation depicted in figure $3(\mathrm{c})$. Therefore, it is necessary to have a counter flow configuration, which leads to a symmetric temperature profile in the lens, optimizing the illumination uniformity. It is also necessary to optimize the channel width. The channel width should be as narrow as possible in order to minimize the fluid flow rate, but wide enough to guarantee an effective heat transfer to the lens.

As operation at temperatures between 800 and $1223 \mathrm{~K}$ is required, a gaseous fluid will be used. In order to have good heat transfer conditions, the fluid pressure must be $\sim 100 \mathrm{kPa}$. 
(a)

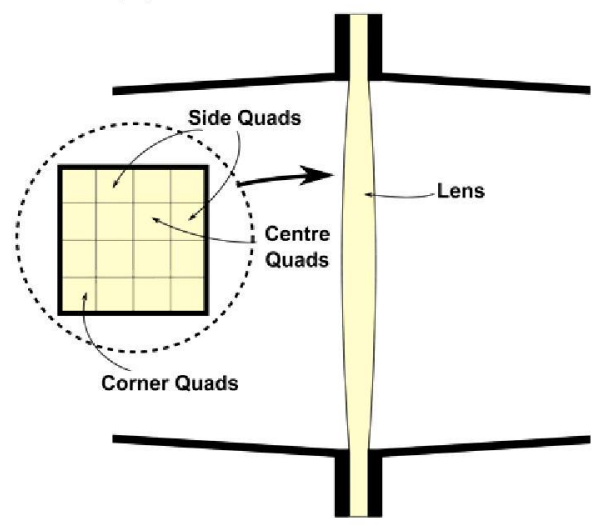

No T. Control

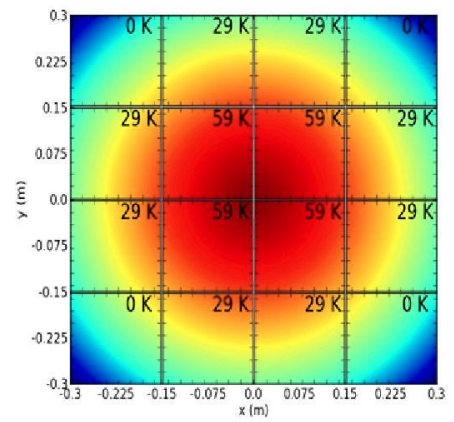

(b)

Heating Resistance
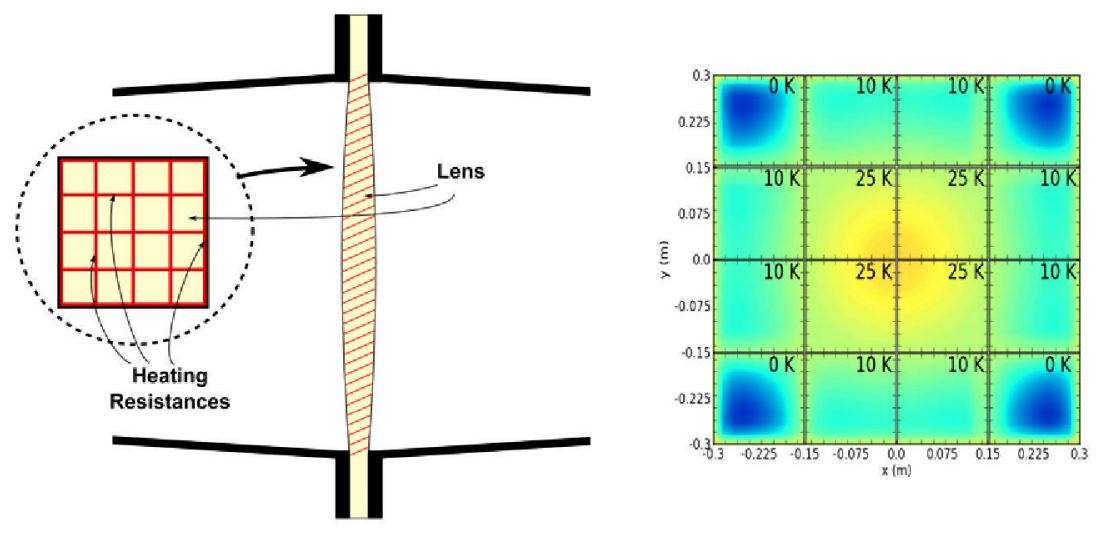

(c)

\section{Heat Transfer Fluid}
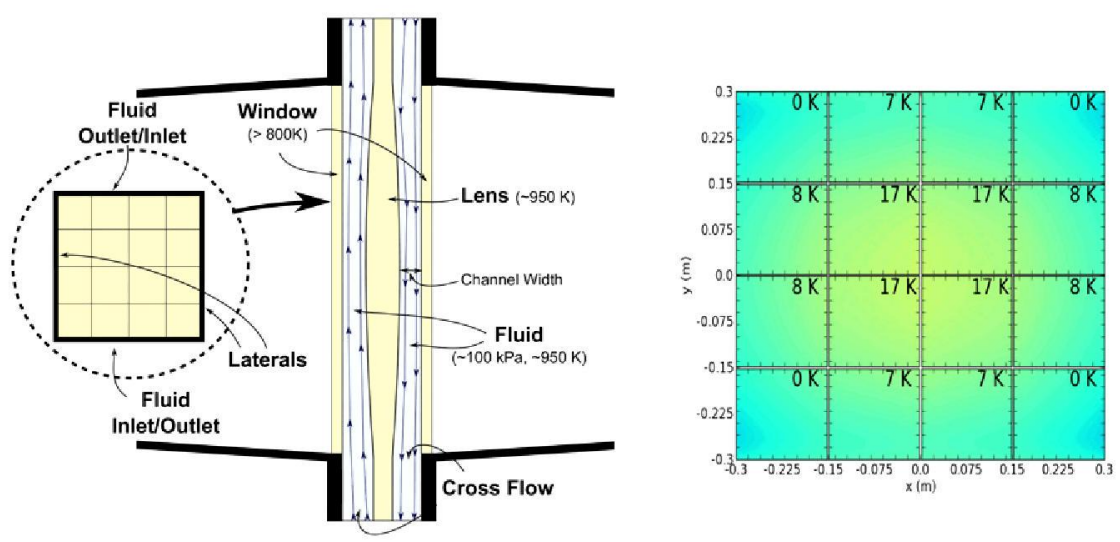

Figure 5. Temperature deviation in relation to the design temperature $\left(T_{0}\right):(a)$ no temperature control system; $(b)$ heating resistances are used between the lens beamlet quadrangles; $(c)$ a heat-transfer fluid is used $\left(\mathrm{CO}_{2}\right.$ with a channel of $6 \mathrm{~mm}$ in the figure).

A pressure drop of $\sim 1 \mathrm{kPa}$ along the channel is enough to set fluid flows able to heat the lens and a fluid temperature of $\sim 950 \mathrm{~K}$ guarantees that both the lens and windows are above $800 \mathrm{~K}$ and the annealing of colour centres is efficient.

The size of the lens and windows is defined by the optical and mechanical requirements. A lens and window size of $60 \times 60 \mathrm{~cm}^{2}$ is defined in order to keep the laser energy density below $10 \mathrm{~J} \mathrm{~cm}^{-2}$. The lens thickness at its borders is $5 \mathrm{~mm}$ in order to hold the lens. Due to the lens curvature radius $(\sim 7.8 \mathrm{~m})$, the lens thickness is $\sim 28 \mathrm{~mm}$ at its centre. The window thickness must be at least $15 \mathrm{~mm}$ to withstand the stresses due to the differential pressure between the fluid $(100 \mathrm{kPa})$ and chamber (vacuum). With this thickness the maximum stress in the window is $\sim 40 \mathrm{MPa}$, below the tensile strength (48 MPa) and the bending stress (67 MPa) [13].

In this work the windows are designed with the minimum thickness that supports the stresses. However if more resistant windows are necessary it is possible to have thicker windows. With thicker windows of $30 \mathrm{~mm}$ thickness, the maximum 
stresses would be reduced to $\sim 10 \mathrm{MPa}$. Instead of having thicker windows it is also possible to add a support grid that strengthens the windows. The support grid can be introduced between the different lens quadrangles in a $4 \times 4$ configuration (similarly to the heating resistances of figure 4). With this solution, the stresses could be reduced to $\sim 3 \mathrm{MPa}$.

The windows do not focus the laser, due to their flatness. There is only a small laser deviation of $\sim 0.1 \mathrm{~mm}$ along the window. This effect is negligible compared to the size of the lens $60 \times 60 \mathrm{~cm}^{2}$. Also, due to the symmetric configuration, this negligible deviation in the first window is corrected in the second window.

The heat-transfer fluid should be transparent to the HiPER laser wavelength $(350 \mathrm{~nm})$, chemically stable in order to prevent reaction with silica at the operational temperatures $(>800 \mathrm{~K})$ and with good heat-transfer properties. Four fluids that fulfil these requirements have been studied: helium, carbon dioxide, argon and xenon. The use of He allows for thin boundary layers and high heat transfer coefficients. However, since He density is low, high volumetric flows are needed. In the case of $\mathrm{CO}_{2}$, the channel width should be slightly larger than for helium. However, as $\mathrm{CO}_{2}$ is heavier, the volumetric flow and pumping power are lower, which can be translated into an easier engineering implementation of the system. Regarding Ar, it presents similarities to $\mathrm{CO}_{2}$, as both have similar densities. However, Ar thermal conductivity and specific heat capacity are lower, which leads to worse cooling properties. Ar could be an alternative to $\mathrm{He}$ or $\mathrm{CO}_{2}$ in case $\mathrm{He}$ permeation or corrosion issues with $\mathrm{CO}_{2}$ posed any problems in the final implementation of the system. Finally Xe, as the heaviest fluid, presents the worst cooling properties. Xe is of interest if the target chamber is to be filled with xenon gas as in the case of some indirect drive target facilities (e.g. the LIFE project). However, for direct target illumination (e.g. the HiPER project), an evacuated chamber is needed and Xe fluid does not present any advantage for a temperature control system. Neutron interaction with the fluid will be much lower than with silica. Due to the lower fluid density and lower thickness of the fluid channel most of the activation will take place in the silica lens and windows. However, the fluid system will have to be controlled to avoid high activation levels or leakages. The transmutation rate for the fluid will be $\sim 100$ appm/fpy.

Silica is quite resistant to corrosion by elements or compounds. Exceptions are some acids, alkalis, alkalineearths and some oxides at high temperature [13]. Therefore, corrosion by the selected fluids will not be a problem, provided the impurity levels are kept under control. Helium solubility and permeation through silica deserve some attention. Helium solubility at $\sim 900 \mathrm{~K}$ is $\sim 5$ appm [25] and the permeation flow through the windows at this temperature is $\sim 10^{13} \mathrm{~cm}^{-2} \mathrm{~s}^{-1}$ [25]. This gives a total of $\sim 2 \times 10^{18}$ atoms s${ }^{-1}$ into the vacuum chamber. This rate is low and can be pumped out by the chamber vacuum system.

Several aspects of system (c), using a heat-transfer fluid, were studied above, and no major problem is expected. In figure 5, a comparison between the different systems (a), (b) and (c) is shown and it is observed that a flat temperature profile can only be obtained with system (c).

For system (a), with no temperature control, we observe in figure $5(a)$ how the neutron irradiation induces a steady temperature profile along the biconvex lenses. The temperature profile has a temperature gradient of $\sim 100 \mathrm{~K}$, which will induce unacceptable aberrations for plant operation. In addition, it will not be possible to maintain a constant temperature during the different regimes of operation making it impossible to pass from start-up to full regime operation. For system (b), with heating resistances, we observe in figure $5(b)$ that due to the low conductivity of silica only a small part of the lens is kept at the desired temperature $\left(T_{0}\right)$. The temperature difference between the coldest part (corners) and the hottest one (lens centre) is $\sim 100 \mathrm{~K}$. Therefore, in order to guarantee a uniform temperature profile, the temperature has to be controlled over the whole lens surface as proposed in system (c). In figure 5(c) we observe how it is possible to have a temperature deviation of only $\sim 20 \mathrm{~K}$ when a heattransfer fluid is used ${ }^{5}$. In the next section, we study how this temperature profile guarantees a focal spot with high illumination uniformity.

\section{Optical performance of the temperature control system}

The focal spot is highly dependent on the temperature profile and a detailed evaluation with realistic temperature profiles is necessary. The proposed fluids ( $\mathrm{He}, \mathrm{CO}_{2}, \mathrm{Ar}$ and $\mathrm{Xe}$ ) were analysed to optimize the channel width in order to improve the figures of merit described in section 2.

Figure 6 shows the uniformity level $(\sigma)$ as a function of channel width for different fluids, while figure 7 shows the efficiency of laser deposition $(\eta)$ during normal operation. In all the cases, the lens design temperature $\left(T_{0}\right)$ is set at the corner temperature ( $\sim 900-950 \mathrm{~K})$. This way, a good illumination uniformity is expected for the foot beamlets, regardless of the fluid or channel width. However, for compression and shock beamlets, the temperature profile may induce aberrations that may lead to poor illumination uniformity.

The shock beamlets pass through the lens centre and the compression beamlets pass through the lens side quadrangles. These quadrangles are at a higher temperature than the corners (foot) due to neutron irradiation and therefore aberrations are introduced. In figures 6 and 7 it is observed how He and $\mathrm{CO}_{2}$ systems with channel widths higher than $\sim 4-6 \mathrm{~mm}$ have high illumination uniformity $(\sigma<1 \%)$ and high efficiency $(\eta \sim 90 \%$ ). For $\mathrm{Ar}$ or $\mathrm{Xe}$, due to their inferior cooling properties, the aberrations are higher than for $\mathrm{He}$ or $\mathrm{CO}_{2}$. This causes a higher level of non-uniformities $(\sigma)$ with lower efficiency $(\eta)$.

From the analysis of the illumination uniformity and efficiency, we conclude that either $\mathrm{He}$ or $\mathrm{CO}_{2}$ could be chosen. However, it is also important to consider that pumping $\mathrm{CO}_{2}$ is easier than pumping $\mathrm{He}$, due to its higher density. For a channel of $6 \mathrm{~mm}, \sim 2.3 \mathrm{~m}^{3} \mathrm{~s}^{-1}$ of He should be pumped out, very high compared to only $\sim 1 \mathrm{~m}^{3} \mathrm{~s}^{-1}$ of $\mathrm{CO}_{2}$. This, in terms of pumping power, translates to $\sim 2.3 \mathrm{~kW}$ for He and $\sim 1 \mathrm{~kW}$ for $\mathrm{CO}_{2}$. In addition, pumping and handling are easier in the case of $\mathrm{CO}_{2}$.

\footnotetext{
5 Only the temperature variation in the lens is considered. Regarding the temperature in the window, its effect on laser focusing is neglected due to the window flatness. Also the temperature variation in the window is low, only $\sim 5 \mathrm{~K}$.
} 

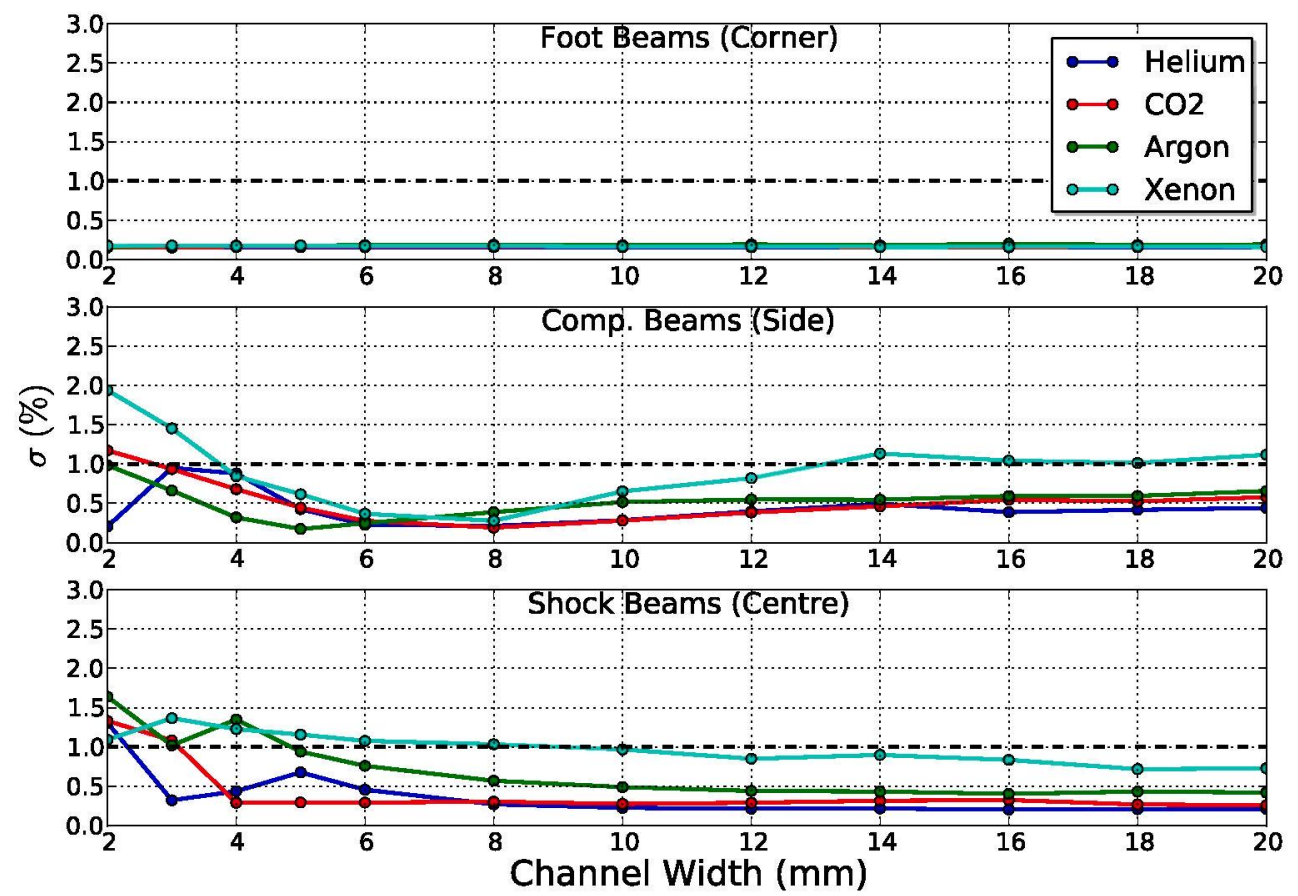

Figure 6. The uniformity level $(\sigma)$ for different heat-transfer fluids as a function of channel widths during normal operation for the different ignition stages (foot, compression and shock).

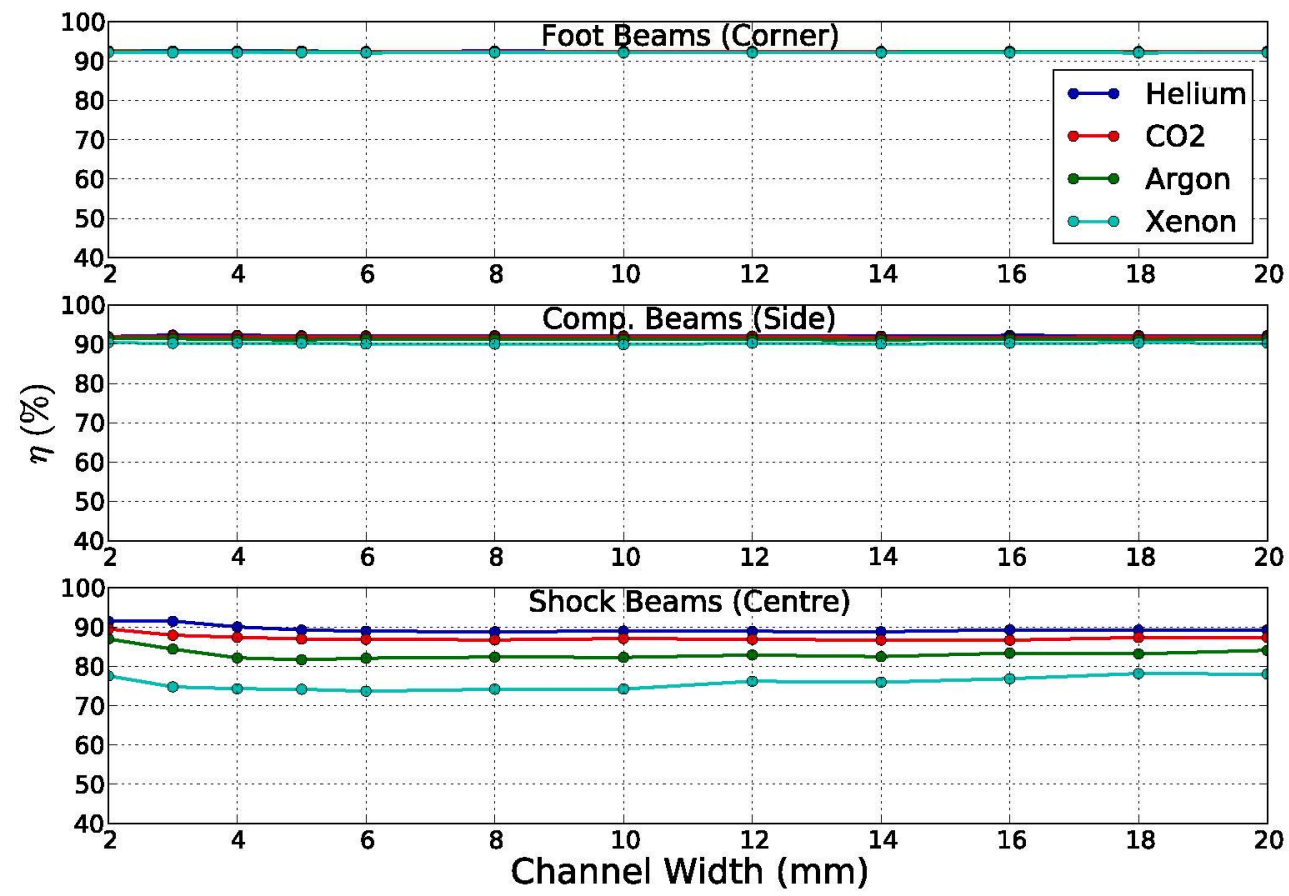

Figure 7. Laser energy deposition efficiency for different heat-transfer fluids and channel widths during normal operation for the different laser beams.

In the previous paragraphs, the illumination uniformity and efficiency at normal operation were analysed. But, what happens during start-up, when there is no neutron irradiation? In normal operation conditions, the lens temperature is defined by the neutron irradiation $\left(510 \mathrm{~kW} \mathrm{~m}^{-3}\right)$ and the heat-transfer fluid (inlet temperature $950 \mathrm{~K}$, pressure drop $1 \mathrm{kPa}$ ). The temperature of the lens is near the design temperature $\left(T_{0}\right)$ and operation of the lens is possible. However, during the start- up there is no neutron irradiation. If the heat-transfer fluid remains at the same conditions as for normal operation, the lens would cool down and would not focus the laser correctly.

Therefore, it is necessary that the fluid transmits more heat to the lens during the start-up until the lens is at its design temperature $\left(T_{0}\right)$. For this purpose the temperature of the fluid is increased. In table 2 we show how by increasing the fluid temperature during the start-up it is possible to maintain a 
Table 2. The main parameters of the final lenses for start-up and normal operation with a channel width of $6 \mathrm{~mm}$

\begin{tabular}{|c|c|c|c|c|c|c|c|c|c|}
\hline \multirow[b]{2}{*}{ Fluid } & \multirow[b]{2}{*}{ Design $T_{0}(\mathrm{~K})$} & \multirow[b]{2}{*}{ Operation regime } & \multirow[b]{2}{*}{ Fluid $T_{\mathrm{f}}(\mathrm{K})$} & \multicolumn{3}{|c|}{$\eta$} & \multicolumn{3}{|c|}{$\sigma$} \\
\hline & & & & Foot & Comp. & Shock & Foot & Comp. & Shock \\
\hline \multirow[t]{2}{*}{$\mathrm{He}$} & \multirow[t]{2}{*}{947} & Start-up & 985 & $92 \%$ & $92 \%$ & $92 \%$ & $0.17 \%$ & $0.18 \%$ & $0.46 \%$ \\
\hline & & Normal & 950 & $92 \%$ & $92 \%$ & $89 \%$ & $0.16 \%$ & $0.23 \%$ & $0.46 \%$ \\
\hline \multirow[t]{2}{*}{$\mathrm{CO}_{2}$} & \multirow[t]{2}{*}{943} & Start-up & 995 & $92 \%$ & $92 \%$ & $92 \%$ & $0.19 \%$ & $0.18 \%$ & $0.18 \%$ \\
\hline & & Normal & 950 & $92 \%$ & $92 \%$ & $87 \%$ & $0.17 \%$ & $0.28 \%$ & $0.29 \%$ \\
\hline \multirow[t]{2}{*}{$\mathrm{Ar}$} & \multirow[t]{2}{*}{926} & Start-up & 1030 & $91 \%$ & $92 \%$ & $92 \%$ & $0.21 \%$ & $0.26 \%$ & $0.41 \%$ \\
\hline & & Normal & 950 & $92 \%$ & $91 \%$ & $82 \%$ & $0.19 \%$ & $0.25 \%$ & $0.76 \%$ \\
\hline \multirow[t]{2}{*}{$\mathrm{Xe}$} & \multirow[t]{2}{*}{909} & Start-up & 1060 & $91 \%$ & $91 \%$ & $92 \%$ & $0.35 \%$ & $0.59 \%$ & $0.53 \%$ \\
\hline & & Normal & 950 & $92 \%$ & $90 \%$ & $74 \%$ & $0.17 \%$ & $0.37 \%$ & $1.08 \%$ \\
\hline
\end{tabular}

good illumination uniformity $(\sigma<1 \%)$ with high efficiencies $(\eta \sim 90 \%)$.

In table 2 , the design temperature $\left(T_{0}\right)$ of the lens is also presented. The design temperature is set to the value of the lens corner temperature at normal operation when the fluid temperature is fixed. The different heat transfer properties of the analysed fluids, $\mathrm{He}$ and $\mathrm{CO}_{2}$, allow for a design temperature in the range 940-950 K, while the design temperatures for $\mathrm{Ar}$ and $\mathrm{Xe}$ are $\sim 930 \mathrm{~K}$ and $\sim 910 \mathrm{~K}$, respectively. Therefore, a higher difference between $T_{0}$ and fluid temperature is required in $\mathrm{Ar}$ and $\mathrm{Xe}$ designs.

The same effect is observed for the fluid temperature during the start-up. In order to keep the lens temperature around $\left(T_{0}\right)$, the fluid temperature is raised from 950 to $\sim 990 \mathrm{~K}$ for $\mathrm{He}$ and $\mathrm{CO}_{2}$, and from 950 to $\sim 1060 \mathrm{~K}$ for $\mathrm{Ar}$ and $\mathrm{Xe}$.

Finally, between start-up and normal operation there will be a transient operation that makes it necessary to adapt the fluid temperature to the neutron irradiation level. Thus, the fluid temperature would be between those corresponding to start-up and normal operation temperatures.

\section{Conclusions}

Throughout this work we have evaluated the neutron irradiation on the HiPER silica transmission final lenses and how the temperature profile affects the laser focusing. We have shown that laser focusing is quite sensitive to temperature variations and thus the temperature has to be carefully controlled during plant operation.

It is important to maintain a high temperature during operation $(>800 \mathrm{~K}$ ) to promote an efficient annealing of colour centres but below the maximum working temperature of silica $(<1223 \mathrm{~K})$. In order to avoid variations in the focal length the temperature must remain constant during operation and uniform along the lens in order to minimize optical aberrations.

A pre-commercial scenario for the HiPER project has been studied. In this scenario final lenses must be placed at a distance of $\sim 16 \mathrm{~m}$. In order to control the temperature a heat-transfer fluid system is proposed. Several fluids have been studied. He and $\mathrm{CO}_{2}$ are adequate to control the lens temperature. $\mathrm{CO}_{2}$ appears to be the best solution due to its cooling properties and easier engineering implementation. The behaviour of the system at normal operation and during start-up has been studied and it is concluded that it is possible to maintain a good illumination uniformity $(\sigma<1 \%)$ and a high efficiency $(\eta \sim 90 \%$ ) by adjusting the fluid temperature.
The main limitations of the proposed solution are the requirement to operate at high temperatures $(>800 \mathrm{~K})$ and the necessity to place the lenses at $16 \mathrm{~m}$ from the target, which results in large facility dimensions. Other solutions may solve the previous limitations at the expense of adding other potential problems. A careful analysis similar to this work must be performed in order to study alternatives to transmission lenses, e.g. Fresnel lenses or GIMM [25].

With the use of Fresnel lenses the size of the facility could be reduced. Fresnel lenses are thinner than transmission lenses and they could be placed nearer the target $(8 \mathrm{~m}, 12 \mathrm{~m})$. However operation at high temperatures is still necessary in order to anneal out colour centres. The focal length variation with temperature is similar for Fresnel and transmission lenses. Therefore, the temperature control system proposed in this work could be also used with Fresnel lenses as the final optical component. Fresnel lenses offer a good alternative to transmission lenses and are in effect the main option for the LIFE project [26]. The main problem of Fresnel lenses stems from the mechanization requirements, which may lead to laser damage and reduced laser focusing capabilities.

To avoid operation at high temperatures, materials different from silica must be used. GIMM could be a solution but its reflectance is highly dependent on the surface quality, which is altered after neutron irradiation. In addition, laser damage is a concern in metal mirrors, as well as, neutron irradiation-induced creep. Further progress in alloys, probably based on the inclusion of nanometer-sized particles, could improve the radiation resistance of the mirrors. Also, with GIMM, large focal lengths $(\sim 24 \mathrm{~m})$ are necessary. Therefore, using GIMM the facility size would be similar or even larger than using biconvex lenses.

Finally, it is important to point out that the presented solution is based on the experimental evidence [22,23] that defects in silica anneal out at high temperatures ( $>800 \mathrm{~K}$ ). However, an unexpected response to irradiation could occur since these results are obtained with neutron fluxes far lower than those expected in HiPER and with different neutron spectra. In addition, the effect of gases (hydrogen, helium) or impurities also requires further research under realistic irradiation conditions, because they may induce laser absorption, or even trigger laser damage. Also the impact of stresses induced by neutron interaction, beam polarization and on wave-front distortion should be studied with an experimental basis. Finally, it must be assessed that the combination of effects will not induce mechanical failure in the system due to creeping, ratcheting or fatigue. 
(a)

$8 \mathrm{~m}-2040 \mathrm{kw} / \mathrm{m}^{3}$
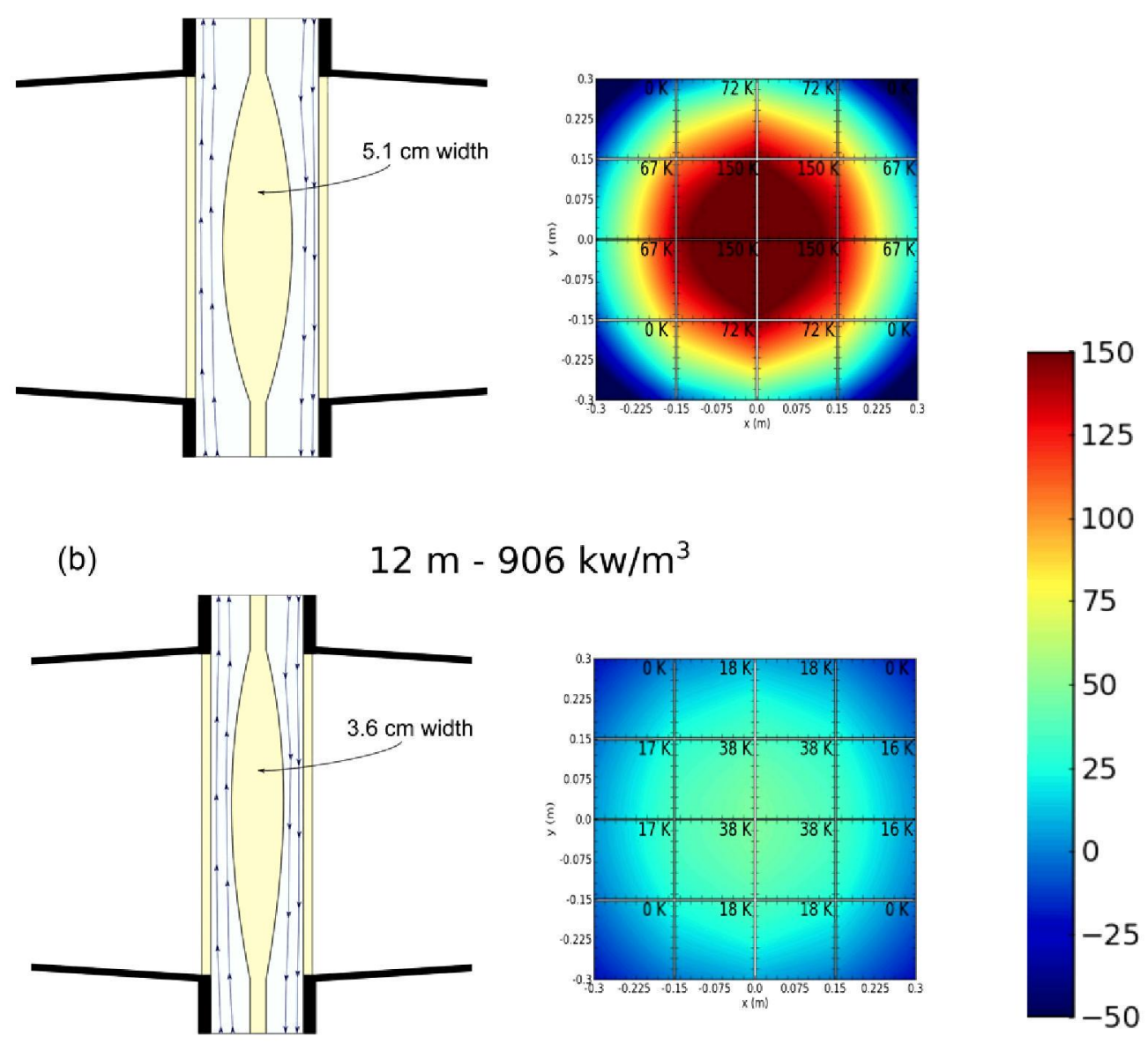

$16 \mathrm{~m}-510 \mathrm{kw} / \mathrm{m}^{3}$

(c)

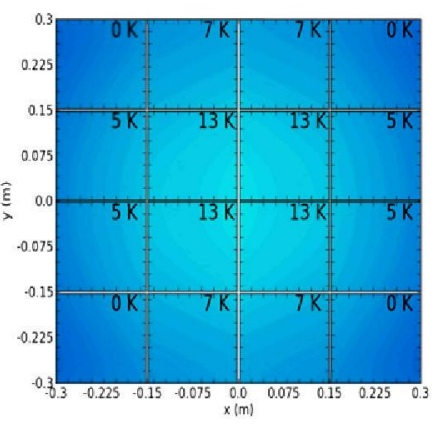

Figure 8. Temperature deviation in relation to the design temperature $\left(T_{0}\right)$. A heat-transfer fluid is used (He with a channel of $20 \mathrm{~mm}$ ). The lens is located at $8 \mathrm{~m}(a), 12 \mathrm{~m}(b)$ and $16 \mathrm{~m}(c)$ for the chamber centre.

\section{Acknowledgments}

We would like to acknowledge the collaboration of Mauro Temporal in fruitful discussions. This work has been possible thanks to the financial support of the Spanish MINECO through the projects AIC-A-2011-0718 (MATFUSLA) and ENE-201239787-C06-03 (RADIAFUS-3) and the PIF program of the Universidad Politécnica de Madrid.

\section{Appendix. Analysis of lenses located nearer the target $(8-12 \mathrm{~m})$}

Throughout this work we have studied the possibility of controlling the temperature of a lens placed at $16 \mathrm{~m}$ from the target. At this distance the pinhole would be placed at $32 \mathrm{~m}$ from the target (see figure 1). A more compact solution would be advantageous. 
Table 3. The optical performance of the lens at different distances for a case using helium and a channel of $20 \mathrm{~mm}$

\begin{tabular}{|c|c|c|c|c|c|c|c|c|c|}
\hline \multirow[b]{2}{*}{ Fluid } & \multirow[b]{2}{*}{$\operatorname{Design} T_{0}(\mathrm{~K})$} & \multirow[b]{2}{*}{ Distance (m) } & \multirow[b]{2}{*}{ Fluid $T_{\mathrm{f}}(\mathrm{K})$} & \multicolumn{3}{|c|}{$\eta$} & \multicolumn{3}{|c|}{$\sigma$} \\
\hline & & & & Foot & Comp. & Shock & Foot & Comp. & Shock \\
\hline \multirow{3}{*}{$\mathrm{He}$} & 918 & 8 & 800 & $78 \%$ & $37 \%$ & $5 \%$ & $2.39 \%$ & $2.32 \%$ & $1.45 \%$ \\
\hline & 926 & 12 & 900 & $92 \%$ & $89 \%$ & $63 \%$ & $0.17 \%$ & $0.58 \%$ & $1.83 \%$ \\
\hline & 950 & 16 & 950 & $93 \%$ & $92 \%$ & $89 \%$ & $0.16 \%$ & $0.46 \%$ & $0.21 \%$ \\
\hline
\end{tabular}

In order to have a conservative solution, we have studied the system using helium with a channel width of $20 \mathrm{~mm}$. As observed in figure 6 , He has the best heat transfer properties (along with $\mathrm{CO}_{2}$ ). In addition, increasing the channel width (beyond $\sim 10 \mathrm{~mm}$ ) shows no further improvement.

In figure 8 , the variation of temperature in relation to $T_{0}$ is shown. It is clearly observed that at $8 \mathrm{~m}$, the temperature variation along the lens is higher than $100 \mathrm{~K}$. Placing the lens at $12 \mathrm{~m}$, the temperature variation is corrected to $\sim 40 \mathrm{~K}$ and placing the lens at $16 \mathrm{~m}$, the temperature variation is less than $15 \mathrm{~K}$. This reduction in the temperature gradient is caused by the lower irradiation and flatter geometry of the lens located further away from the target.

In table 3, the performance of the lens is shown and it is observed that with the lenses at $16 \mathrm{~m}$ the efficiency is high ( $\eta \sim 90 \%$ ) and the illumination uniformity is $\operatorname{good}(\sigma<1 \%$ ). However if the lens is placed at $8 \mathrm{~m}$ the illumination uniformity is not acceptable $(\sigma>1 \%)$. When placing the lens at $12 \mathrm{~m}$ an intermediate scenario is found, where the illumination uniformity is good $(\sigma<1 \%)$ for the foot and compression beams and worse for the shock beams $(\sigma>1 \%)$.

In this work it is concluded that it is possible to place the lenses at $16 \mathrm{~m}$ from the target with the temperature control systems proposed. At the expense of increasing the nonuniformities at the end of the ignition (shock beams), it would be possible to place the lenses at $\sim 12 \mathrm{~m}$ from the target. Finally the lenses cannot be placed much nearer $(\sim 8 \mathrm{~m})$ as the nonuniformities would increase for all the ignition stages.

\section{References}

[1] Brereton S. 2013 Overview of the National Ignition Facility Health Phys. 104 544-56

[2] Latkowski J.F., Abbott R.P., Aceves S., Anklam T., Badders D., Cook A.W., DeMuth J., Divol L., El-Dasher B. and Farmer J.C. 2011 Chamber design for the laser inertial fusion energy (LIFE) engine Fusion Sci. Technol. 60 54-60

[3] Le Garrec B., Novaro M., Tyldesley M., Juarez R., Sanz J., Perlado M., Rus B., Collier J. and Edwards C. 2011 HiPER laser reference design Proc. SPIE $808080801 \mathrm{~V}$

[4] Tillack M.S. and Pulsifer J.E. 2009 Long-term survival of grazing-incidence metal mirrors for laser fusion Fusion Sci. Technol. 56446

[5] Di Nicola P. et al 2012 Beam and target alignment at the National Ignition Facility using the target alignment sensor (TAS) Proc. SPIE 8505 85050B

[6] Garoz D., González-Arrabal R., Juárez R., Álvarez J., Sanz J., Perlado J.M. and Rivera A. 2013 Silica final lens performance in laser fusion facilities: HiPER and LIFE Nucl. Fusion $\mathbf{5 3} 013010$

[7] Rivera A., Garoz D., Juarez R., Alvarez J., González-Arrabal R. and Perlado J.M. 2011 Lifetime of silica final lenses subject to HiPER irradiation conditions Proc. SPIE $791679160 \mathrm{~S}$
[8] Ribeyre X., Schurtz G., Lafon M., Galera S. and Weber S. 2009 Shock ignition: an alternative scheme for HiPER Plasma Phys. Control. Fusion 51015013

[9] Atzeni S., Davies J.R., Hallo L., Honrubia J.J., Maire P.H., Olazabal-Loumé M., Feugeas J.L., Ribeyre X., Schiavi A. and Schurtz G. 2009 Studies on targets for inertial fusion ignition demonstration at the HiPER facility Nucl. Fusion 49055008

[10] Canaud B. and Garaude F. 2005 Optimization of laser-target coupling efficiency for direct drive laser fusion $\mathrm{Nucl}$. Fusion 45 L43

[11] Bercegol H., Boscheron A., Di-Nicola J.-M., Journot E., Lamaignère L., Něauport J. and Razě G. 2008 Laser damage phenomena relevant to the design and operation of an ICF laser driver J. Phys.: Conf. Ser. 112032013

[12] Le Garrec B., Di-Nicola J.M. and Beau V. 2007 Métrologie des faisceaux et de la tache focale de la LIL J. Physique IV 138 297-307

[13] Heraeus Quarzglas Quartz Glass for Optics: Data and Properties (http://heraeus-quarzglas.com/media/ webmedia_local/downloads/broschren_mo/ DataandProperties_Optics_fusedsilica.pdf)

[14] Páramo A.R., Sordo F., Perlado J.M. and Rivera A. 2014 Viability of the ESS-Bilbao neutron source for irradiation of nuclear fusion materials $J$. Nucl. Mater. 444 469-74

[15] Temporal M. and Canaud B. 2011 Stochastic homogenization of the laser intensity to improve the irradiation uniformity of capsules directly driven by thousands laser beams Eur. Phys. J. D 65 447-51

[16] Schiavi A., Atzeni S. and Marocchino A. 2011 Illumination stability for high-repetition-rate laser facilities in direct-drive inertial confinement fusion Europhys. Lett. 9435002

[17] Pelowitz D.B. 2005 MCNPXTM User's Manual

[18] Chdwick M.B. et al 2011 ENDF/B-VII.1 Nuclear data for science and technology: cross sections, covariances, fission product yields and decay Nucl. Data Sheets 112 2887-996

[19] Raffray A.R., Haynes D. and Najmabadi F. 2003 IFE chamber walls: requirements, design options, and synergy with MFE plasma facing components $J$. Nucl. Mater: 313-316 23-31

[20] Fluent Inc. 2006 Fluent 6.3 User's Guide

[21] Bell I., Quoilin S. and Wronski J. http://coolprop.sourceforge.net/

[22] Marshall C.D., Speth J.A. and Payne S.A. 1997 Induced optical absorption in gamma, neutron and ultraviolet irradiated fused quartz and silica J. Non-Cryst. Solids $21259-73$

[23] Latkowski J.F., Payne S.A., Kubota A., Caturla M.J., Dixit S.N. and Speth J.A. 2003 Fused silica final optics for inertial fusion energy: radiation studies and system-level analysis Fusion Sci. Technol. $\mathbf{4 3} 540-58$

[24] Wray J.H. and Neu J.T. 1969 Refractive index of several glasses as a function of wavelength and temperature $J$. Opt. Soc. Am. 59 774-6

[25] Swets D.E., Lee R.W. and Frank R.C. 1960 Diffusion coefficients of helium in fused quartz $J$. Chem. Phys. $3417-22$

[26] Bayramian A. et al 2011 Compact, efficient laser systems required for laser inertial fusion energy Fus. Sci. Technol. $6028-48$ 